\title{
Spontaneous displacement of a pacemaker electrode and its subsequent successful reimplantation
}

\author{
JOHN GIALAFOS, ANASTASIOS THEOPHILIS, GEORGE SIAMAS \\ From B Department of Cardiology and Cardiac Department, University of Athens Medical School, \\ Hippokration Hospital, Athens, Greece
}

SUMMARY A case of what was assumed to be twiddler's syndrome was caused by spontaneous twisting and displacement of the electrode on the fortieth postoperative day. The pacemaker had not been manipulated by the patient, the pocket was tight, and the proximal electrode was well secured. The complication was managed successfully by reimplanting the same electrode after stiffening the lead near the generator with a portion of the stylet.

Pacemaker twiddler's syndrome was first described by Bayliss et al in $1968 .^{1}$ They reported a case in which the pulse generator had twisted in its subcutaneous pocket, apparently because it had been manipulated by the patient. This twisting led to retraction of the electrode into the superior vena cava and consequent interruption of pacing. Since then, there have been several reports of similar cases with various access routes (transvenous, epicardial), ${ }^{2-4}$ types of leads (unipolar, bipolar), ${ }^{3}$ and pulse generator pocket positions (pectoral, abdominal) ${ }^{34}$ and comparisons of conventional pacemakers and implantable internal automatic cardioverter defibrillators. $^{5}$

We describe a patient in whom the transvenous ventricular pacing electrode became displaced by spontaneous twising of the electrode. We managed this complication in a new way.

\section{Case report}

A 68 year old woman (weight $67 \mathrm{~kg}$, height $1.61 \mathrm{~m}$ ) underwent implantation of a Medtronic 5941 VVI pacemaker with target tip unipolar electrode (Medtronic-4011-58) because of symptomatic 2:1 atrioventricular block. The pacing threshold was $0.4 \mathrm{~V}$ or $0.8 \mathrm{~mA}$ with $450 \Omega$ resistance and satisfactory endocardial potentials. The electrode was attached to the surrounding tissue with three double silk sutures (Mersilik 3.5), according to the standard practice of our pacing unit. The position and functioning of the pacing system were entirely satisfactory initially and on the second examination 20 days later (fig 1a).

Requests for reprints to Dr John Gialafos, 17-19 Ploutarchou Street, 10675 Athens, Greece.
On the fortieth postoperative day, however, the patient was brought to the clinic because of loss of pacing capture. The $x$ ray (fig $1 \mathrm{~b}$ ) showed that the electrode tip was displaced into the superior vena cava and that the lead was twisted near to the generator. The position and orientation of the generator were almost exactly as they had been at the time of implantation. The patient consistently denied touching or manipulating the area of the pacemaker pocket.

On reoperation, the pacemaker pocket was found to be tight around the generator but there were several twists in the electrode lead close to the generator, despite the double sutures still being in place. Because it was difficult to withdraw the electrode past the junction of the subclavian and cephalic veins, we decided on a novel solution to the problem. We reimplanted the same electrode, after inserting approximately half of the soft stylet $(35 \mathrm{~cm})$ into the part of the lead nearest the generator. Thus the distal part of the stylet, which we had curved slightly to assume its final position, was in the superior vena cava, while the proximal end coincided with the proximal end of the electrode, which was screwed into the generator. (Cutting the stylet with scissors had left a slight flange around what became the proximal end, and this prevented the stylet sliding towards the electrode tip.) In this way, the proximal part of the electrode was stiffened and made more resistant to twisting (fig 2).

\section{Discussion}

As first described by Bayliss et al. ${ }^{1}$ the lead displacement in pacemaker twiddler's syndrome is due to a capstan effect produced by the pulse generator's 


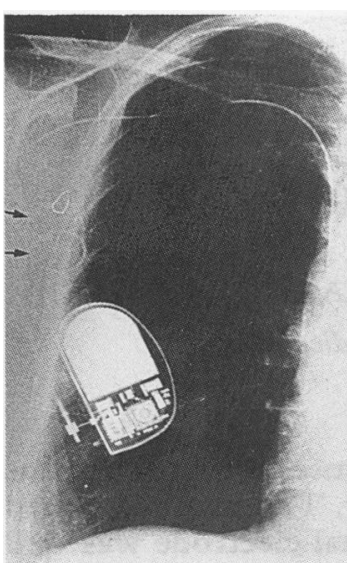

(a)

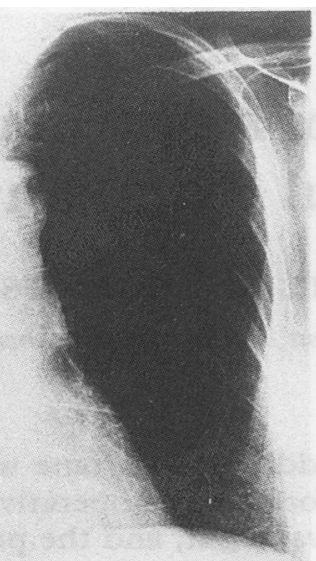

(b)

Fig 1 (a) Radiograph showing the satisfactory position of the pacing system. Note the two loops in the electrode lead (arrows). (b) Radiograph showing displacement of the electrode tip to the superior vena cava and twisting of the electrode (arrow) close to the pacemaker. The position and orientation of the generator are almost identical with those shown in $(a)$.

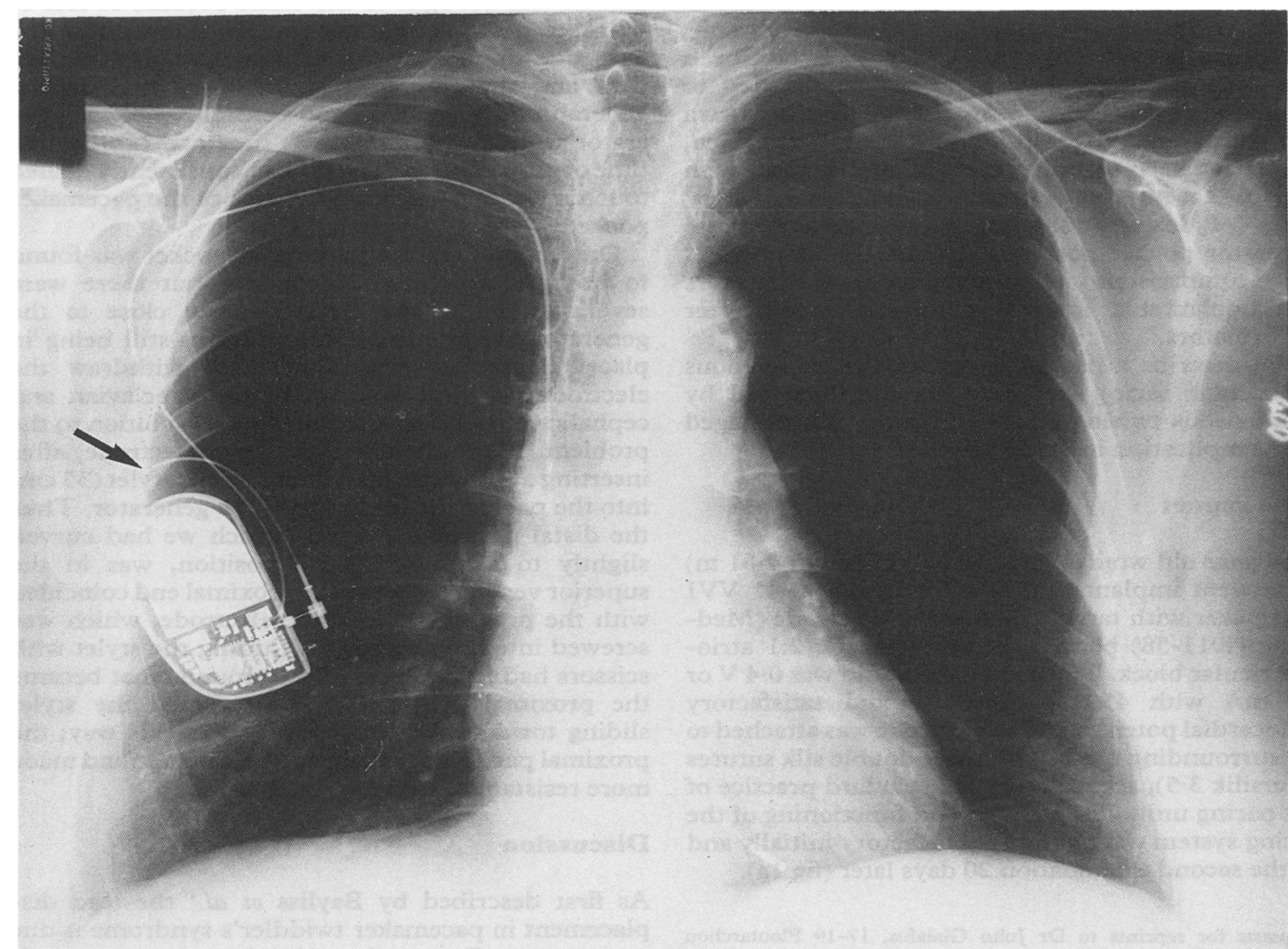

Fig 2 Radiograph after reimplantation of the electrode in the apex. The proximal part of the electrode forms one large loop (arrow) because of its extra stiffness, in contrast with the two loops formed by the original, more flexible electrode, as shown in fig $1 a$.

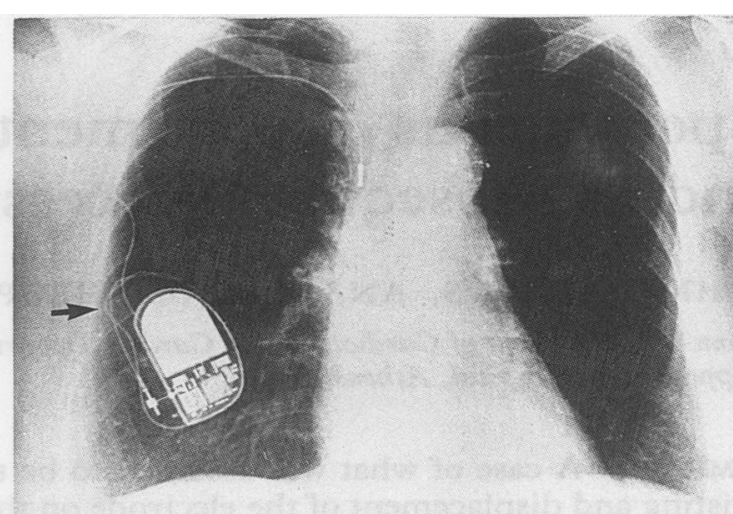


rotation within a capacious pulse generator pocket. According to Smyth and Millette, this syndrome is found in obese patients, in whom a loose generator pocket tends to form. ${ }^{6}$ Spontaneous rotation of the pulse generator may then occur, as a result of arm movement, when there is a loose pocket, when the generator has not been fixed firmly in place, or when a single fixation suture has been used, which then provides a fulcrum around which the pulse generator can rotate.

In our case the patient was not obese, there was no slackness in the generator pocket, and the patient consistently denied fiddling with the pocket.

The $x$ ray showed that the pulse generator was in almost exactly the same position as on implantation. On reoperation, we found that the pocket fitted tightly around the generator, with no fluid accumulation, and the three double securing sutures were clearly identifiable.

We concluded that the electrode was dislodged by spontaneous twisting, which retracted the electrode. This case provides strong grounds for believing that the term "pacemaker twiddler's syndrome" is not always apt. We agree with Smyth and Millette ${ }^{6}$ that this term should be abandoned.

It is generally believed that when a pacemaker lead is disturbed in this way it should be removed and replaced or fixed and replaced by a new lead. ${ }^{6}$ In our patient it was impossible to remove the original electrode. Because we strongly believed that the lead twisting was spontaneous and that the lead itself was undamaged, we decided to reimplant the same electrode, leaving a large part of the stylet inside the lead and connected with it to the generator. The stylet is quite flexible and we did not expect that the end of the stylet would damage the lead, especially as the stylet was shaped appropriately before insertion. In this way we stiffened the electrode as far as the superior vena cava, sufficiently to preclude further twisting. On reimplantation, the threshold was only $0.3 \mathrm{~V}$ or $0.7 \mathrm{~mA}$ with $472 \Omega$ resistance, confirming that the electrode was in good condition. The pacemaker was not given any extra support.

The follow up over eight months has been uneventful, with no signs of lead damage or displacement, and the patient is doing well.

\section{References}

1 Bayliss CE, Beanlands DS, Baird RJ. The pacemakertwiddler's syndrome: a new complication of implantable transvenous pacemakers. Can Med Assoc J 1968;99:371-3.

2 Stamford W, Coyle FL, Dooley BN, et al. Transvenous pacemaker failure: migration of catheter lead by patient manipulation. Ann Thorac Surg 1969;5:162-4.

3 Meyer JA, Fruehan CT, Delmonico JE. The pacemaker twiddler's syndrome-a further note. $J$ Thorac Cardiovasc Surg 1974;67:903-7.

4 Guharay BN, Ghose JC, Majumdar H, Basu AK. The pacemaker-twiddler's syndrome: another disadvantage of abdominal implantation of pulse generators. $\mathrm{Br}$ J Surg 1977;64:655-60.

5 Veltri EP, Mower M, Read PR. Twiddler's syndrome: a new twist. PACE 1984;7:1004-9.

6 Smyth NPD, Millette ML. Complications of pacemaker implantation. In: Barold SS, ed. Modern cardiac pacing Mount Kisco, New York: Futura, 1985: 257-75. 